\section{Dual geometric active contour for image segmentation}

Guopu Zhu*, Qingshuang Zeng, and Changhong Wang Harbin Institute of Technology, Space Control and Inertial Technology Research Center, Harbin 150001, China E-mail: zgp@ @it.edu.cn

\begin{abstract}
A dual geometric active contour applied mainly in image segmentation is proposed. Two contours of the dual geometric active contour evolve from the interior and exterior of the segmented object, respectively, to the desired boundary. The inner and outer contours represented by level set functions interact with each other during the evolutions of the active contour to avoid getting in local minima traps of the functional optimizations. The experiments on image segmentation show that the proposed dual geometric active contour can relieve the problem in initialization. () 2006 Society of Photo-Optical Instrumentation Engineers.

[DOI: 10.1117/1.2333566]
\end{abstract}

Subject terms: active contours; image segmentation; level set function.

Paper 060289LR received Apr. 14, 2006; revised manuscript received Jun. 14, 2006; accepted for publication Jun. 20, 2006; published online Aug. 25, 2006.

\section{Introduction}

Active contours have been widely researched and applied in image processing and computer vision since they were first introduced by Kass et al. ${ }^{1}$ The applications of active contours range from image segmentation and shape modeling to visual tracking, and so on. This letter mainly addresses the application of active contours in image segmentation. Generally speaking, active contours can be classified into two kinds: parametric active contours ${ }^{1-3}$ and geometric active contours. ${ }^{4-8}$ Parametric active contours represented by point sets or B-splines are efficient in computation. On the contrary, geometric active contours represented by level set functions are a vast computational task, but they have the advantage of automatically handling changes in topology over parametric active contours. Unfortunately, both kinds of active contours suffer difficulties in choosing the appropriate initial contours. A dual active contour ${ }^{2}$ was proposed by Gunn and Nixon to relieve the problem in initialization for a parametric active contour. Inspired by their work, in this letter, we correspondingly propose a dual geometric active contour, of which the inner and outer contours evolve respectively from the interior and exterior of the segmented object to the desired boundary. The two contours interact with each other to avoid getting in local minima traps of the functional optimizations of the active contour during the evolutions of the level set functions that represent the contours. Promising results on image segmentation demonstrate the potentials of the proposed dual geometric active contour.

\footnotetext{
${ }^{*}$ Corresponding author. Present address: P. O. Box 3015, Harbin Institute of Technology, Science Park, No. 2 Yikuang Street, Harbin, 150001, China 0091-3286/2006/\$22.00 @ 2006 SPIE
}

\section{Dual Geometric Active Contour}

We implement the dual geometric active contour on ChanVese active contour, which is a typical geometric active contour. Given a 2-D gray-value image $f: \Omega \rightarrow \mathbb{R}^{+}$. ChanVese active contour ${ }^{8}$ as a closed dynamic curve $C$ is defined to minimize an energy functional, which is given by

$$
\begin{aligned}
E_{\mathrm{CV}}\left(u_{1}, u_{2}, \phi\right)= & \mu \int_{\Omega}\left|\nabla H_{\epsilon}(\phi)\right| \mathrm{d} x+\int_{\Omega}\left(f-u_{1}\right)^{2} H_{\epsilon}(\phi) \\
& +\left(f-u_{2}\right)^{2}\left[1-H_{\epsilon}(\phi)\right] \mathrm{d} x,
\end{aligned}
$$

where $C$ is represented by the zero-level set of a level set function $\phi: \Omega \rightarrow \mathbb{R}$, and $H_{\epsilon}(\bullet)$ parametrized with $\epsilon$ is a smooth approximation of the Heaviside function. ${ }^{8}$ The first term on the right-hand side of Eq. (1) measures the length of the curve $C$. And the second term, in which $u_{1}$ and $u_{2}$ are the mean gray values of the image $f$, respectively, inside and outside of the curve $C$, measures the homogeneities in the different regions. The positive parameter $\mu$ weights the relative importance of the two terms.

As mentioned, active contours suffer difficulties in choosing the appropriate initial contours. Inspired by the work of Gunn and Nixon, ${ }^{2}$ we correspondingly propose a dual geometric active contour implemented on a Chan-Vese active contour. The proposed active contour consists of two contours initialized simultaneously inside and outside the object boundary. The inner and outer contours, which are represented by the level set functions $\phi$ and $\psi$, respectively, are defined to minimize the following energy functional

$$
\begin{aligned}
E_{\mathrm{DCV}}\left(u_{1}, u_{2}, \phi ; v_{1}, v_{2}, \psi\right)= & E_{\mathrm{CV}}\left(u_{1}, u_{2}, \phi\right)+E_{\mathrm{CV}}\left(v_{1}, v_{2}, \psi\right) \\
& +\tau \int_{\Omega}\left[H_{\epsilon}(\phi)-H_{\epsilon}(\psi)\right]^{2} \mathrm{~d} x,
\end{aligned}
$$

where the new parameters $v_{1}$ and $v_{2}$ denote the mean gray values of the image $f$, respectively, inside and outside of the curve represented by the zero-level set of $\psi$. The second term on the right-hand side of Eq. (2) is called the interaction term, which is defined to reduce the difference between the inner and outer contours. Through the interaction between the two contours, the active contour will quite likely avoid getting in local minima traps of the functional optimizations. The positive constant $\tau$ is the weight parameter for the interaction term. Note that although the interaction term in the dual geometric active contour has a form similar to the shape term in the shape-based active contour proposed in literature, ${ }^{9}$ the principles underlying the two terms are greatly different.

Minimizing energy functional $E_{\mathrm{DCV}}$ with respect to $\phi$ and $\psi$, respectively, we can obtain two associated EulerLagrange equations. Then, the evolutions of the level set functions $\phi$ and $\psi$ are implemented by the following gradient descents:

$$
\begin{aligned}
\frac{\partial \phi}{\partial t}= & \delta_{\epsilon}(\phi)\left\{\mu \operatorname{div}\left(\frac{\nabla \phi}{|\nabla \phi|}\right)-\left[\left(f-u_{1}\right)^{2}-\left(f-u_{2}\right)^{2}\right]\right. \\
& \left.-2 \tau\left[H_{\epsilon}(\phi)-H_{\epsilon}(\psi)\right]\right\},
\end{aligned}
$$

and 


\section{OE LETTERS}

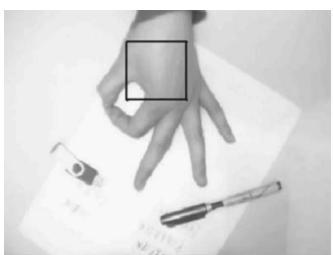

(a)

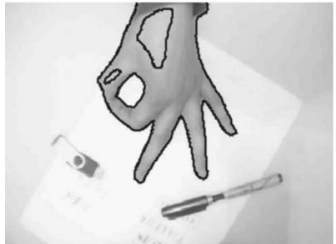

(d)

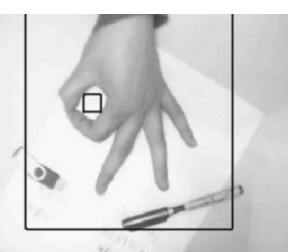

(b)

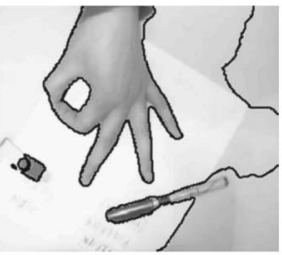

(e)

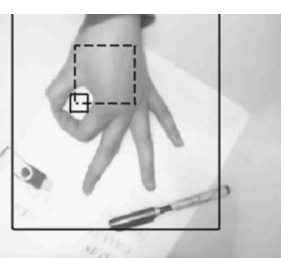

(c)

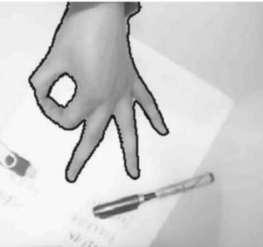

(f)

Fig. 1 Segmentation of the hand image: (a), (b), and (c) initializations of Chan-Vese active contour respectively form the inside and outside of the hand boundary as well as the initialization of the dual geometric active contour and (d), (e), and (f) the corresponding segmental results at the steady state.

$$
\begin{aligned}
\frac{\partial \psi}{\partial t}= & \delta_{\epsilon}(\psi)\left\{\mu \operatorname{div}\left(\frac{\nabla \psi}{|\nabla \psi|}\right)-\left[\left(f-v_{1}\right)^{2}-\left(f-v_{2}\right)^{2}\right]\right. \\
& \left.-2 \tau\left[H_{\epsilon}(\psi)-H_{\epsilon}(\phi)\right]\right\},
\end{aligned}
$$

where $\delta_{\epsilon}(\bullet)=H_{\epsilon}^{\prime}(\bullet)$ is the approximation of the delta function, and $t$ denotes the artificial time for parametrizing the gradient descent. Note that $H_{\epsilon}(\phi)$ and $H_{\epsilon}(\psi)$ in Eqs. (3) and (4) can also be, respectively, replaced by $\phi$ and $\psi$ in the practical implementation. ${ }^{10}$ The parameters $u_{1}$ and $u_{2}$, and $v_{1}$ and $v_{2}$ required by the computation of the two gradient descents are updated by

$u_{1}=\frac{\int f(x) H_{\epsilon}(\phi) \mathrm{d} x}{\int H_{\epsilon}(\phi) \mathrm{d} x}, \quad u_{2}=\frac{\int f(x)\left[1-H_{\epsilon}(\phi)\right] \mathrm{d} x}{\int\left[1-H_{\epsilon}(\phi)\right] \mathrm{d} x}$,

and $v_{1}=\frac{\int f(x) H_{\epsilon}(\psi) \mathrm{d} x}{\int H_{\epsilon}(\psi) \mathrm{d} x}, \quad v_{2}=\frac{\int f(x)\left[1-H_{\epsilon}(\psi)\right] \mathrm{d} x}{\int\left[1-H_{\epsilon}(\psi)\right] \mathrm{d} x}$,

with the evolutions of the level set functions $\phi$ and $\psi$.

Based on the preceding description of the proposed active contour, we summarize the complete algorithm as follows:

Step 1. Initialize the level set functions $\phi$ and $\psi$.

Step 2. Compute $u_{1}$ and $u_{2}$, and $v_{1}$ and $v_{2}$ by Eqs. (5) and (6), respectively.

Step 3. Evolve $\phi$ according Eq. (3), then redistance it.

Step 4. Evolve $\psi$ according Eq. (4), then redistance it. Go to step 2 .

Note that the preceding process proceeds until the evolutions of the level set functions $\phi$ and $\psi$ have converged.

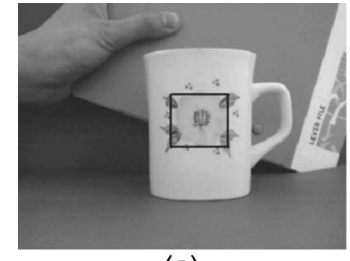

(a)

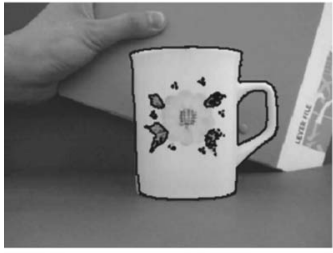

(d)

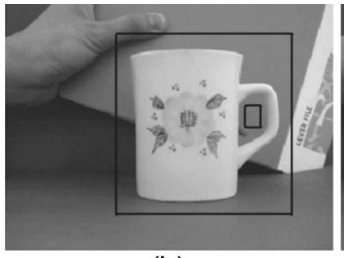

(b)

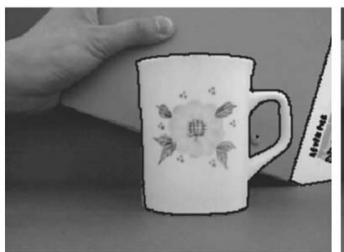

(e)

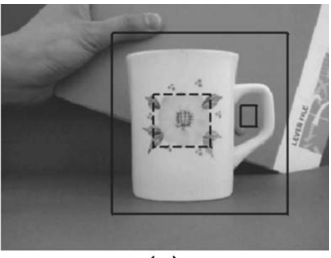

(c)

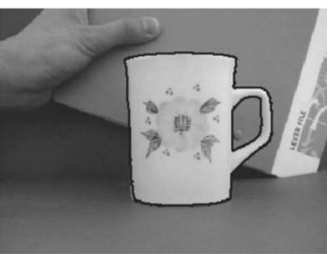

(f)

Fig. 2 Segmentation of the cup image: (a), (b), and (c) initializations of Chan-Vese active contour respectively form the inside and outside of the cup boundary as well as the initialization of the dual geometric active contour and (d), (e), and (f) the corresponding segmental results at the steady state. 


\section{Experimental Results}

We validated the performance of the dual geometric active contour on many real images by comparing it with the Chan-Vese active contour. In all our experiments, we use $\epsilon=0.05, \mu=0.005 \times 255^{2}$, and $\Delta t=0.02$ (the step of the artificial time $t$ ) for both Chan-Vese active contour and the proposed active contour, and we use $\tau=2.5 \times 255^{2}$ especially for the proposed active contour.

Figure 1 shows an example of our experiments. The size of the tested hand image is $320 \times 240$ pixels. Figure 1 (a) shows the initialization of the Chan-Vese active contour from the inside of the hand boundary, and Fig. 1(d) shows its corresponding result at the steady state. It is shown that the contour at the steady state excludes two regions that belong to the hand area, since the gray values of the pixels in the two regions are closer to the mean gray value of the image in the background than that in the hand area. Contrarily, Fig. 1(b) shows the initialization of the Chan-Vese active contour from the outside of hand boundary. As shown in Fig. 1(e), the contour at the steady state includes several undesired regions in which the gray values of the pixels are closer to the mean gray value of the image in the hand area, such as the regions of the pen and the USB disk. In Fig. 1(c), a dual geometric active contour is initialized, and the solid lines denote the initialization of the outer contour and the dashed lines denote the initialization of the inner contour. Figure 1(f) shows that the two contours completely superpose on each other and exactly conform to the hand boundary at the steady state.

Similarly, another example of our experiments is shown in Fig. 2. The size of the tested cup image is 320 $\times 240$ pixels. It is also shown that the dual geometric active contour outperforms the Chan-Vese active contour in the segmentation.

\section{Conclusions}

We proposed a dual geometric active contour to overcome the difficulties in initialization that are suffered by the con- ventional geometric active contours. The dual geometric active contour simultaneously initializes two contours, respectively, inside and outside of desired boundary. And the two contours interact with each other in their evolving procedures to avoid the local optimizations of the energy functional. The experiments conducted on real images show that the proposed dual active contour is robust to bad contour initializations, while it is still, to some extent, dependent on the choice of the initial contour. In the future work, a shape prior can be incorporated into the proposed active contour model to further relieve the problem in initialization.

\section{Acknowledgments}

The authors wish to thank the anonymous reviewers and associate editor Giordano Beretta for their useful comments.

\section{References}

1. M. Kass, A. Witkin, and D. Terzopoulos, "Snakes: active contour models," Int. J. Comput. Vis. 1, 321-331 (1988).

2. S. R. Gunn and M. S. Nixon, "A robust snake implementation: a dual active contour," IEEE Trans. Pattern Anal. Mach. Intell. 19, 63-68 (1997).

3. C. Xu and J. L. Prince, "Snakes, shapes, and gradient vector flow," IEEE Trans. Image Process. 7, 359-369 (1998).

4. V. Caselles, F. Catte, T. Coll, and F. Dibos, "A geometric model for active contours in image processing," Numer. Math. 66, 1-31 (1993).

5. R. Malladi, J. A. Sethian, and B. C. Vemuri, "Shape modeling with front propagation: a level set approach," IEEE Trans. Pattern Anal. Mach. Intell. 17, 158-175 (1995).

6. S. Kichenesamy, A. Kumar, P. Olver, A. Tannenbaum, and A. Yezzi, "Conformal curvature flows: from phase transitions to active contours," Arch. Ration. Mech. Anal. 134, 275-301 (1996).

7. V. Caselles, R. Kimmel, and G. Sapiro, "Geodesic active contours," Int. J. Comput. Vis. 22, 61-79 (1997).

8. T. Chan and L. Vese, "Active contours without edges," IEEE Trans. Image Process. 10, 266-277 (2001).

9. T. Chan and W. Zhu, "Level set based shape prior segmentation," Proc. IEEE Int. Conf. on Computer Vision and Pattern Recognition (CVPR), pp. 1164-1170, Washington, DC (2005).

10. D. Cremers, N. Sochen, and C. Schnörr, "A multiphase dynamic labeling model for variational recognition-driven image segmentation," Int. J. Comput. Vis. 66, 67-81 (2006). 